\title{
Anti-proliferative activity of $A$. Oxyphylla and its bioactive constituent nootkatone in colorectal cancer cells
}

\author{
Eunsu Yoo', Jaehak Lee ${ }^{1}$, Pattawika Lertpatipanpong ${ }^{1}$, Junsun Ryu², Chong-Tai Kim ${ }^{3}$ Eul-Yong Park ${ }^{3}$ and \\ Seung Joon Baek ${ }^{1 *}$ (iD
}

\begin{abstract}
Background: A. oxyphylla extract is known to possess a wide range of pharmacological activites. However, the molecular mechanism of A. oxyphylla and its bioactive compound nootkatone in colorectal cancer is unknown.

Methods: Our study aims to examine the role of $A$. oxyphylla and its bioactive compound nootkatone, in tumor suppression using several in vitro assays.

Results: Both A. oxyphylla extract and nootkatone exhibited antiproliferative activity in colorectal cancer cells. A. oxyphylla displayed antioxidant activity in colorectal cancer cells, likely mediated via induction of HO-1.

Furthermore, expression of pro-apoptotic protein NAG-1 and cell proliferative protein cyclin D1 were increased and decreased respectively in the presence of A. oxyphylla. When examined for anticancer activity, nootkatone treatment resulted in the reduction of colony and spheroid formation. Correspondingly, nootkatone also led to increased NAG-1 expression and decreased cyclin D1 expression. The mechanism by which nootkatone suppresses cyclin D1 involves protein level regulation, whereas nootkatone increases NAG-1 expression at the transcriptional level. In addition to having PPARY binding activity, nootkatone also increases EGR-1 expression which ultimately results in enhanced NAG-1 promoter activity.
\end{abstract}

Conclusion: In summary, our findings suggest that nootkatone is an anti-tumorigenic compound harboring antiproliferative and pro-apoptotic activity.

Keywords: Nootkatone, NAG-1, Cyclin D1, A. oxyphylla

\section{Background}

Alpinia oxyphylla belongs to the Zingiberaceae family and is widely cultivated in Asia as one of the most frequently used plant extracts in oriental medicine. The most well-known medicinal effect of $A$. oxyphylla includes enhancing the internal and astringent activities of the kidney and spleen [1]. Recent studies have shown

\footnotetext{
* Correspondence: baeksj@snu.ac.kr

'Department of Veterinary Medicine, College of Veterinary Medicine and Research Institute for Veterinary Science, Seoul National University, Seoul 08826, South Korea

Full list of author information is available at the end of the article
}

that A. oxyphylla possesses a wide range of pharmacological activities, such as anti-diabetes [2], anti-fibrosis [3], anti-diarrheal [4], and anti-cancer [5].

A. oxyphylla contains various chemical constituents, including essential oils, sesquiterpenes, flavones, diarylheptanoids, glycosides and steroids. Amongst them, nootkatone is one of the more abundant components [6]. Nootkatone has also been identified as the main fragrant component of grapefruit with a wide range of beneficial effects including anti-inflammation activities [7], AMPK activation [8] and neuroprotective effects [9]. Other bioactive compounds in A. oxyphylla,

C C The Author(s). 2020 Open Access This article is licensed under a Creative Commons Attribution 4.0 International License, which permits use, sharing, adaptation, distribution and reproduction in any medium or format, as long as you give appropriate credit to the original author(s) and the source, provide a link to the Creative Commons licence, and indicate if changes were made. The images or other third party material in this article are included in the article's Creative Commons licence, unless indicated otherwise in a credit line to the material. If material is not included in the article's Creative Commons licence and your intended use is not permitted by statutory regulation or exceeds the permitted use, you will need to obtain permission directly from the copyright holder. To view a copy of this licence, visit http://creativecommons.org/licenses/by/4.0/. The Creative Commons Public Domain Dedication waiver (http://creativecommons.org/publicdomain/zero/1.0/) applies to the data made available in this article, unless otherwise stated in a credit line to the data. 
yakuchinone A [10] and yakuchinone B [11], are also known to have several biological activities including anti-cancer activity. However, the molecular target of nootkatone and other bioactive compounds in cancer or in cell proliferation is unknown.

By analyzing identified molecular targets of phytochemicals, we discovered that the nonsteroidal antiinflammatory drug [NSAID]-activated gene-1 (NAG-1, also known as GDF15) is highly induced by several phytochemicals [12]. Ectopic expression of NAG-1 causes cell growth arrest, and overexpression of NAG-1 in human colon cells results in reduced tumor formation in the nude mouse model [13]. Although in vitro assays show contradictory results, studies conducted in NAG-1 TG and NAG-1 KO mice consistently demonstrate a clear association between NAG-1 expression and tumor suppression [14]. Thus, NAG-1 induction is a likely molecular mechanism for anticancer activity induced by phytochemicals.

Cyclin D1, another common target of phytochemicals, is often overexpressed in various cancer cell types and tumors. In addition to its role in the cell cycle, cyclin D1 functions as a critical regulator of DNA repair, and thus constitutes a key molecular regulator of transcription [15]. A large number of anticancer chemicals have been shown to downregulate cyclin D1 in various cancer cell types by triggering multiple signaling pathways [16].

EGR-1 is induced very early in the apoptotic process, and mediates the activation of downstream regulators such as p53 [17]. However, EGR-1-induced apoptosis has also been reported in $\mathrm{p} 53^{-/-}$cells, indicating the existence of both p53-dependent and p53-independent pathways. EGR-1 also activates tumor suppressor gene phosphatase and tensin homolog (PTEN) during UV irradiation and suppresses the growth of transformed cells both in soft agar and in athymic nude mice [18]. While these results indicate that EGR-1 plays a significant role in growth suppression, the consequences of EGR-1 expression may vary depending on the cellular context. Discrepancies in its role may depend on expression levels of other EGR-1 family members, Sp1, EGR-1 binding repressors, or other factors yet to be identified. Interestingly, EGR-1 has been linked to increased NAG-1 promoter activity, mediated by nonsteroidal antiinflammatory drugs [19].

Thus, NAG-1, cyclin D1, or EGR-1 could be a molecular target of many bioactive compounds that could lead to anti-proliferation activity. The identification of the molecular target of nootkatone may lead to the development of better single compounds for cancer therapy.

In this study, we identified the biological activity of $A$. oxyphylla and its major compound nootkatone as an inducer of the pro-apoptotic protein NAG-1 and a suppressor of cyclin D1, thereby inhibiting cell proliferation in colon cancer cells. Further, the mechanism by which nootkatone affects cyclin D1 and NAG-1 has been studied. Our results indicated that EGR-1 plays a pivotal role in nootkatone-induced NAG-1 expression, while the proteosomal degradation pathway contributes to nootkatone-mediated cyclin D1 downregulation.

\section{Methods \\ Reagents}

A. oxyphylla was purchased from Kyung-Dong Market in Seoul, Korea. The authenticity was confirmed at least twice through morphological analysis by Dr. Jaeyoon Cha, Department of Food Science and Nutrition, Dong-A University, Busan, Republic of Korea. A voucher specimen (No. EHNP-H8) has been deposited in the R\&D Center, EastHill Corporation, Suwon, Gyeonggi-do, Republic of Korea. The plants were washed and ground using a laboratory mill to a particle size of 100 mesh. Ethanol (70\%) was added to the ground plants and extracted at $70^{\circ} \mathrm{C}$ for $48 \mathrm{~h}$ with stirring at $500 \mathrm{rpm}$. The extract was filtered using Toyo No. 4 filter paper and concentrated using a vacuum evaporator. Finally, the concentrate was diluted in dimethyl sulfoxide to obtain a final concentration $100 \mathrm{mg} / \mathrm{mL}$. Nootkatone was purchased from Tokyo Chemical Industry (Tokyo, Japan). Epoxomicin and Puromycin (P8833-10) were purchased from Sigma Aldrich (St. Louis, MO, USA) and MG132 was purchased from AdooQ ${ }^{\circ}$ Bioscience (Irvine, CA, USA). Antibodies for Cyclin D1 (sc-753), HRP conjugated $\beta$-actin (sc-47,778), and p53 (sc-126) were purchased from Santa Cruz Biotechnology (Dallas, TX, USA). Antibody for NAG1 was previously described [13].

\section{Cell culture}

All cells used in this study were purchased from American Type Culture Collection (ATCC). Cells were tested by ATCC for post-freeze viability, growth properties, morphology, mycoplasma contamination, species determination (cytochrome c oxidase I assay and short tandem repeat analysis), sterility test and human pathogenic virus testing. Upon arrival, cell lines were straightaway resuscitated and frozen in aliquots in liquid nitrogen. HCT-116 (Human colorectal carcinoma) and HT-29 (Human colorectal adenocarcinoma) cells were cultured using McCoy's 5A media (Gibco life technologies, Carlsbad, CA, USA). SW480 (Human colorectal adenocarcinoma), DLD-1 (Human colorectal adenocarcinoma) were cultured using RPMI-1640 media (GIBCO). Both McCoy's 5A media and RPMI-1640 media contained 10\% Fetal bovine serum (FBS; GIBCO) and 1\% penicillin/ streptomycin (GIBCO). All cells were maintained at $37^{\circ} \mathrm{C}$ and $5 \% \mathrm{CO}_{2}$. 


\section{Plasmid transfection and luciferase assay}

The promoter-luciferase constructs pNAG1-1086/+41, pNAG1-474/+41, and pNAG1-133/+41 were previously described [12]. The expression vector pcDNAEGR-1 has also been described [19]. The luciferase and pRL-null plasmids were transfected into cells using PolyJet $^{\text {ts }}$ In Vitro DNA Transfection reagent (SignaGen, Frederick, MD, USA) according to manufacturer's instructions. Luciferase activity was measured using DualLuciferase $^{\oplus}$ Reporter Assay kit (Promega, WI, USA) as previously described [20].

\section{Colony formation assay}

Both HCT-116 and SW480 cells were seeded at a density of $1 \times 10^{4}$ cells/well in 6 -well culture plates. Cells were treated with nootkatone at various doses $(10 \mu \mathrm{M}$, $50 \mu \mathrm{M}$, or $100 \mu \mathrm{M}$ ) for 9 days. The culture media containing indicated concentrations of nootkatone were changed every 3 days. After treatment, the plate was washed with phosphate-buffered saline, and cells were fixed with $4 \%$ paraformaldehyde (Biosesang, Gyeonggido, Korea), followed by staining with $1 \%$ crystal violet solution (V5265, Sigma Aldrich). The number of colonies was counted using Image J software $1.52 \mathrm{a}(\mathrm{Na}-$ tional Institutes of Health, MD, USA).

\section{Western blot analysis}

Western blot analysis was conducted as previously described [21]. Briefly, $50 \mu \mathrm{g}$ proteins were separated using $12 \%$ sodium dodecyl sulfate-polyacrylamide gel electrophoresis and transferred to a nitrocellulose membrane (GVS filter technology, Zola Predosa BO, Italy). The blotted membrane was then blocked with 5\% skim milk for $1 \mathrm{hr}$ at room temperature and incubated overnight with specific antibodies at $4{ }^{\circ} \mathrm{C}$. After incubation with HRP conjugated secondary antibody in 5\% skim milk for $1 \mathrm{hr}$ at room temperature, the blotted membranes were visualized using the Alliance Q9 mini imaging system (Cambridge, UK) and quantified using ImageJ software 1.52a (National Institutes of Health, MD, USA).

\section{RNA isolation and reverse transcription polymerase chain} reaction (RT-PCR)

Total RNA was isolated using TRIzol reagent (Invitrogen, Carlsbad, CA, USA). Five hundred nanograms of total RNA was used to synthesize cDNA using Verso cDNA Synthesis kit (Thermo scientific, Waltham, MA, USA). PCR products were amplified using the following primer pairs: cyclin D1 (F: 5'-CAA TGA CCC CGC ACG ATT TC-3', R: 5' ${ }^{\prime}$ AAG TTG TTG GGG CTC CTC AG-3'), NAG-1 (F: 5' -CTC CAG ATT CCG AGA CTT GC-3', R:5'-AGA CAT ACG CAG GTG CAG GT-3'), GAPDH (F: 5'-GAC CAC AGT CCA TGC CAT CAC T-3', R: TCC ACC ACC CTG TTG CTG
TAG-3'). Thermal cycling conditions for NAG-1 were as follows: initial denaturation at $95^{\circ} \mathrm{C}$ for $2 \mathrm{~min}$, followed by $25-35$ cycles of $94{ }^{\circ} \mathrm{C}$ for $30 \mathrm{~s}, 53.2^{\circ} \mathrm{C}$ for 30 s, and $72{ }^{\circ} \mathrm{C}$ for $1 \mathrm{~min}$, and final elongation at $72{ }^{\circ} \mathrm{C}$ for 5 min. For cyclin D1 and GAPDH, amplification and annealing temperatures were set to $52.5^{\circ} \mathrm{C}$ and $60{ }^{\circ} \mathrm{C}$ respectively. PCR products were electrophoresed on a $1.5 \%$ agarose gel and photographed using the Alliance Q9 mini imaging system.

\section{Cell proliferation assay}

HCT-116 and SW480 were seeded in 96-well plates $(1 \times$ $10^{3}$ cells/well for HCT-116 cells and $2 \times 10^{3}$ cells/well for SW480 cells) and incubated for $24 \mathrm{~h}$ with $100 \mu \mathrm{L}$ of complete medium. Different dose of $A$. oxyphylla was treated for the indicated time. Cell proliferation assays were then performed using CellTiter $96^{\circ}$ AQueous One Solution (Promega, WI, USA) according to the manufacturer's instructions. After indicated time of culture, $20 \mu \mathrm{L}$ of One Solution reagent was added to each well and cells were incubated for $1 \mathrm{~h}$ at $37^{\circ} \mathrm{C}$. Cell viability was estimated by measuring the absorbance at $492 \mathrm{~nm}$ using Multiskan FC spectrophotometer (Thermo Fisher Scientific, Waltham, MA).

\section{Antioxidant activity assay}

The 2,2-diphenyl-1-picrylhydrazyl (DPPH, \#14805, Cayman Chemical, MI, USA) and 2,2 -azino-bis(3-ethylbenzothiazoline-6-sulfonic acid) (ABTS, \#A1888, Sigma Aldrich) were used for the radical scavenging assay, as previously described [22]. The absorbance was measured using a Multiskan ${ }^{\mathrm{TM}}$ FC microplate photometer (Thermo Fisher Scientific, Waltham, MA). L-ascorbic acid (\#A0537, TCI, Tokyo, Japan) was used as a reference standard in both assays. Determination of the percentage of radical scavenging effect was considered using the following equation:

$$
\begin{gathered}
\% \text { Inhibition }=100-[(\text { Absorbance of sample }- \text { Absorbance of blank }) \\
\times 100 / \text { Absorbance of control }] .
\end{gathered}
$$

The VCEAC for ABTS assay and the IC50 value were calculated as half the concentration of the sample that can scavenge $50 \%$ of the DPPH free radical.

\section{Spheroid assay}

Seven hundred and fifty HCT-116 cells were seeded in an ultra-low attachment round bottom 96-well plate (Coster, Kennebunk, ME, USA), and cultured for 4 days. After spheroids were formed, half of the media was replaced with complete media containing 2 times the required dosage of nootkatone, and spheroids were incubated for 3 days. Spheroid viability was measured by the CellTiterGlo ${ }^{\circledR}$ D Cell Viability Assay (Promega, Madison, WI, 
USA) in accordance with the manufacturer's instuctions. Spheroid volume was calculated using the following for-

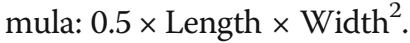

\section{Statistics}

Data are expressed as mean \pm SD from at least three independent experiments. Statistical analyses were performed using one-way ANOVA test. All comparisons are relative to untreated or carrier controls and significant differences have been indicated as ${ }^{*} p<0.05 ;{ }^{* *} p<0.01 ;{ }^{* * *} p<0.001$.

\section{Results}

\section{Effects of A. oxyphylla extract on cell growth}

A. oxyphylla has been used as a traditional Chinese medicine for many years. However, the molecular mechanism of $A$. oxyphylla extract as an anticancer agent has not been elucidated. In the current study, ethanol extracts of $A$. oxyphylla have been obtained and examined for potential antiproliferative activity in two human colorectal cancer cell lines. Treatment with A. oxyphylla extract affected HCT-116 and SW480 cell growth in a dose- and time-dependent manner with IC50 values of $89.3 \mu \mathrm{g} / \mathrm{ml}$ and $>100 \mu \mathrm{g} / \mathrm{ml}$, respectively. (Fig. 1a-b). At $48 \mathrm{~h}$ and $96 \mathrm{~h}, A$. oxyphylla significantly inhibited cell growth both in p53 wild type (HCT-116) and p53 mutant colorectal cancer cells (SW480), at a concentration of $100 \mu \mathrm{g} / \mathrm{ml}$.

\section{Antioxidant activity of $A$. oxyphylla extracts}

Antioxidant activity of natural compounds has been shown to be highly related to anticancer effects [23]. Thus, the antioxidant activity of $A$. oxyphylla extract has been investigated. DPPH and ABTS radical scavenging activity assays were performed to determine the antioxidant capacity of $A$. oxyphylla extract at various concentrations. The effects of $A$. oxyphylla extract and ascorbic acid on the ABTS radical compound are shown in Fig. 2a. The ABTS scavenging activities increased in correlation with increasing concentrations of $A$. oxyphylla extract. As a positive control, L-ascorbic acid displayed high antioxidant activity with decreased ABTS radical at a low concentration. Similarly, the DPPH radical scavenging activity of $A$. oxyphylla extract is presented in Fig. 2b. Scavenging activity increased in a dose-dependent manner up to $1000 \mathrm{ng} / \mathrm{mL}$; with a similar trend observed in the ABTS assay. Antioxidant activity was also measured using a luciferase construct containing the antioxidant response element (ARE). After transfection into HCT-116 cells, luciferase activity was increased in the presence of $A$. oxyphylla, suggesting that $A$. oxyphylla may activate NRF2 which binds ARE sites (Fig. 2c). In addition, Heme oxygenase-1 (HO-1) protein levels were analyzed by western blot as a marker for antioxidant activity to confirm the antioxidant effect of the extract. Results showed that $\mathrm{HO}-1$ protein levels increased in a dose-dependent manner (Fig. 2d). Overall, our results indicate that $A$. oxyphylla possesses antioxidant activity.

\section{NAG-1 and cyclin D1 expression in the presence of $A$. oxyphylla}

To elucidate the molecular mechanism by which $A$. oxyphylla affects anticancer activity in colorectal cancer cells, expression of NAG-1 and cyclin D1 have been determined. An increase in NAG-1 expression was observed, whereas cyclin D1 expression level was decreased
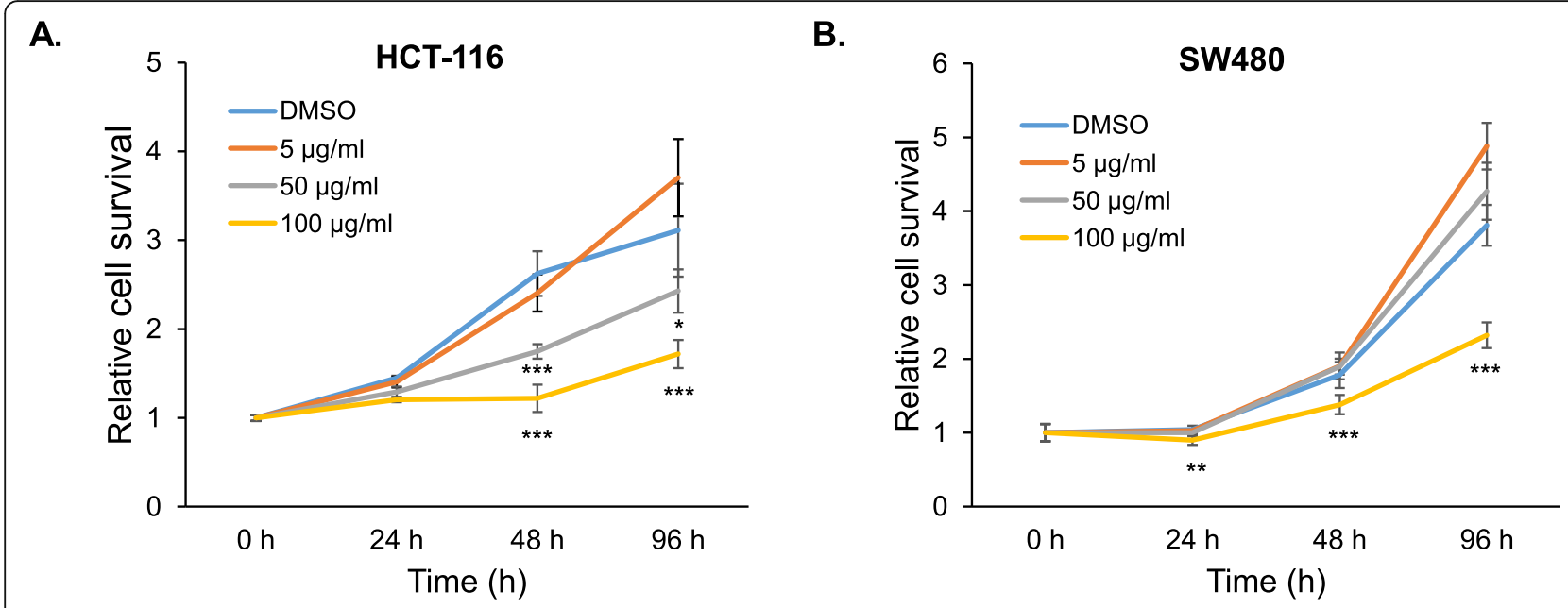

Fig. 1 Proliferation assay of colorectal cancer cells in the presence of A. oxyphylla. a HCT-116 and b SW480 cells were treated with various concentrations of $A$. oxyphyllal at different time points. Cell numbers were measured by the Cell Proliferation Assay (Promega) after adding an indicated dose of $A$. oxyphylla. DMSO was used as a control. The results from five independent experiments are shown as mean \pm SD with statistical significance displayed as ${ }^{*} p<0.05,{ }^{* * *} p<0.001$, compared to DMSO-treated cells 


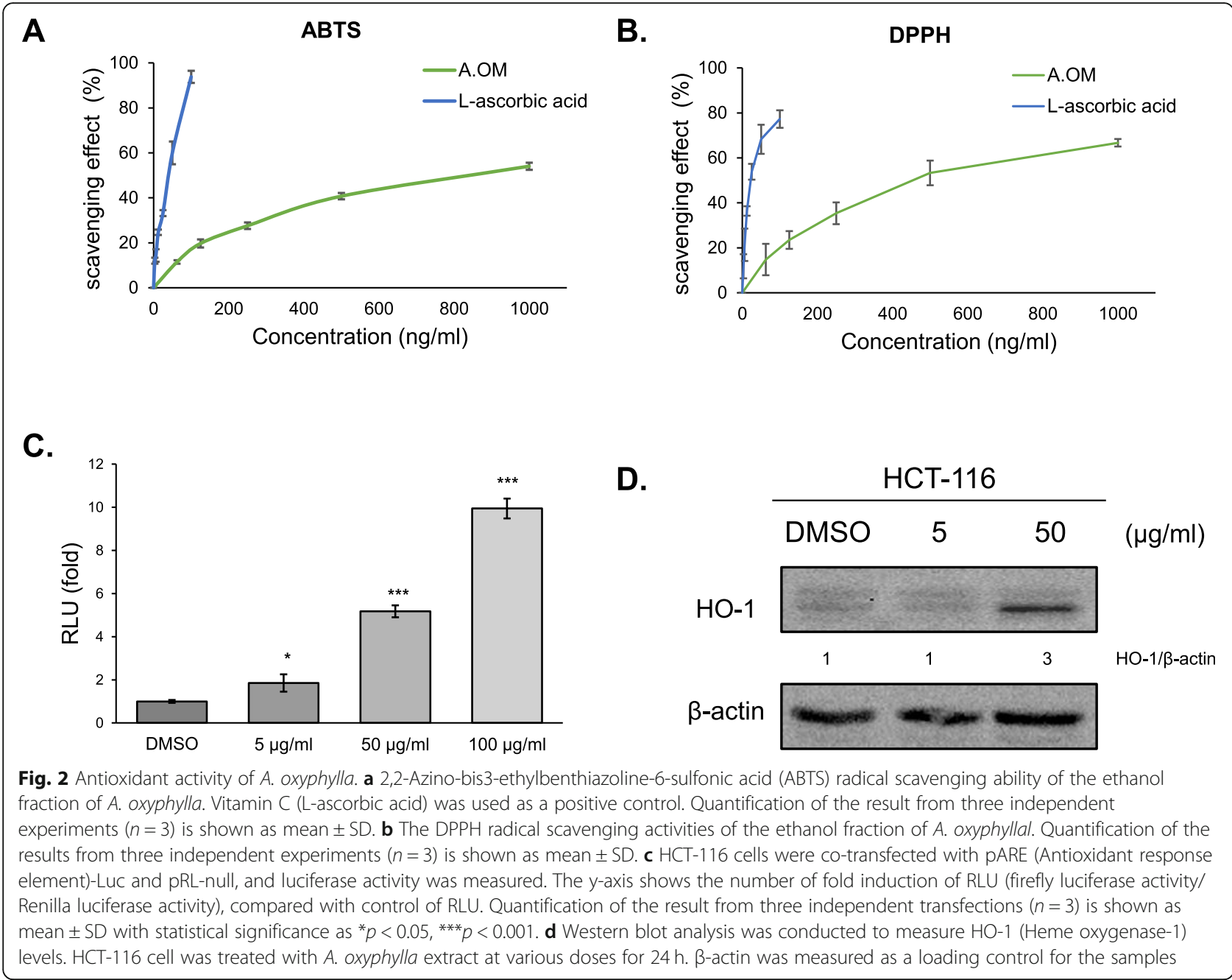

in all four colorectal cancer cell lines treated with $A$. oxyphylla (Fig. 3a-d). These results suggest that $A$. oxyphylla extract may regulate colorectal cancer cell growth by elevating NAG-1 protein expression and decreasing cyclin D1 protein expression.

\section{Nootkatone exhibits anti-tumorigenic activity in colorectal cancer cells}

One of the bioactive compounds found in $A$. oxyphylla is nootkatone. (Fig. 4a) [6]. To determine whether nootkatone can account for the antiproliferative effect of $A$. oxyphylla, we analyzed cell growth both by counting cells and by performing colony and spheroid formation assays. Colorectal cancer cells were treated with nootkatone at concentrations of $10 \mu \mathrm{M}, 50 \mu \mathrm{M}$ and $100 \mu \mathrm{M}$, wherein nootkatone treatment resulted in cell growth inhibition in a dose and time dependent manner (Fig. 4b), similar to the trend observed following $A$. oxyphylla extract treatment (Fig. 1). Furthermore, in the colony formation assay, nootkatone showed a dose-dependent inhibition of colony formation in two colorectal cancer cell lines (Fig. 4c). In the spheroid formation assay, nootkatone decreased spheroid formation (viability and volume) in HCT-116 cells (Fig. 4d), indicating that nootkatone possesses anti-tumorigenic activity not only in a $2 \mathrm{D}$ culture system, but also in a 3D culture system.

\section{Nootkatone decreases cyclin D1 and increases NAG-1 expression}

We examined whether nootkatone may decrease cyclin D1 or increase NAG-1 expression at the transcription level. An increased RNA level of NAG-1 was observed in the presence of nootkatone treatment, whereas cyclin D1 RNA levels did not change in cells treated with nootkatone (Fig. 5a). Protein levels of NAG-1 and cyclin D1 were also analyzed revealing that both NAG-1 and cyclin D1 were altered by nootkatone treatment at the protein level (Fig. 5b). Taken together, our results indicate that nootkatone may affect cyclin D1 at the protein level and NAG-1 at the transcriptional level. 


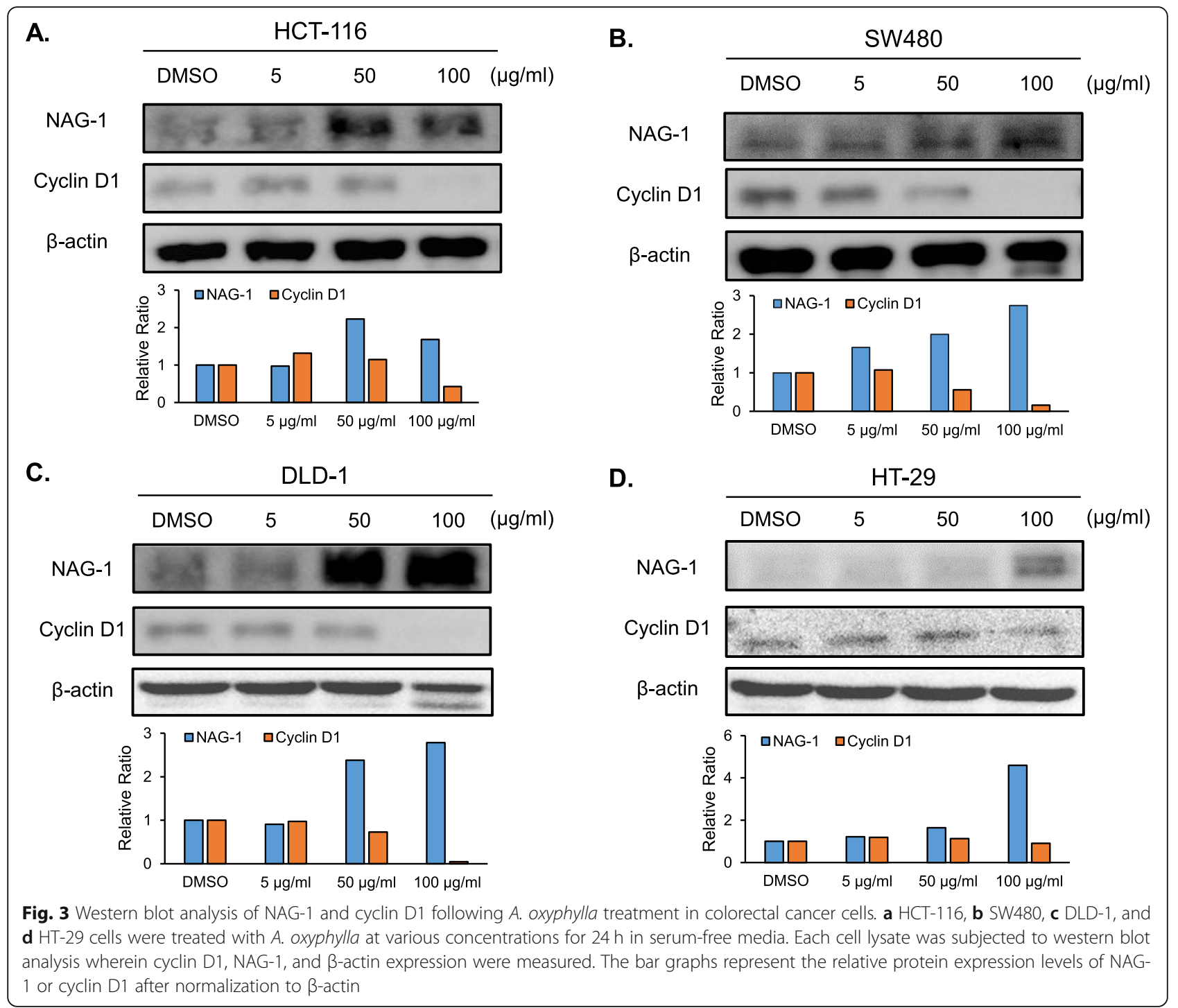

\section{Nootkatone decreases cyclin D1 expression via proteosomal pathway}

To clarify the molecular mechanism by which nootkatone decreases cyclin D1 protein levels, a protein stability assay was performed wherein HCT-116 and SW480 cell lines were treated with puromycin and proteosomal inhibitors. In both cell lines, cyclin D1 protein expression was dramatically decreased by nootkatone treatment compared to the control, indicating that nootkatone may affect the stability of the cyclin D1 protein in the cells (Fig. 6a). Furthermore, proteosomal inhibitors epoxomicin and MG132 were combined with nootkatone to determine whether this would rescue the protein-destabilizing effect of cyclin D1 by nootkatone. Results showed that epoxomicin and MG132 partially restored cyclin D1 protein levels, indicating that nootkatone may affect cyclin D1 degradation through a proteosomal degradation pathway-related mechanism (Fig. 6b).

\section{Nootkatone increases transcriptional expression of NAG-1 via EGR-1}

To examine the direct effect of nootkatone on NAG-1 expression, a NAG-1 promoter-luciferase construct containing $1086 \mathrm{bp}$ was transfected into HCT-116 cells and luciferase activity was measured following nootkatone treatment. NAG-1 promoter activity was increased in a dose-dependent manner, with the highest expression corresponding to nootkatone treatment at a concentration of $100 \mu \mathrm{M}$ (Fig. 7a). To identify the response element region in the NAG-1 promoter which responded to nootkatone, three deletion mutant clone plasmids were designed and analyzed for nootkatone-inducing activity following transfection. The response element position 


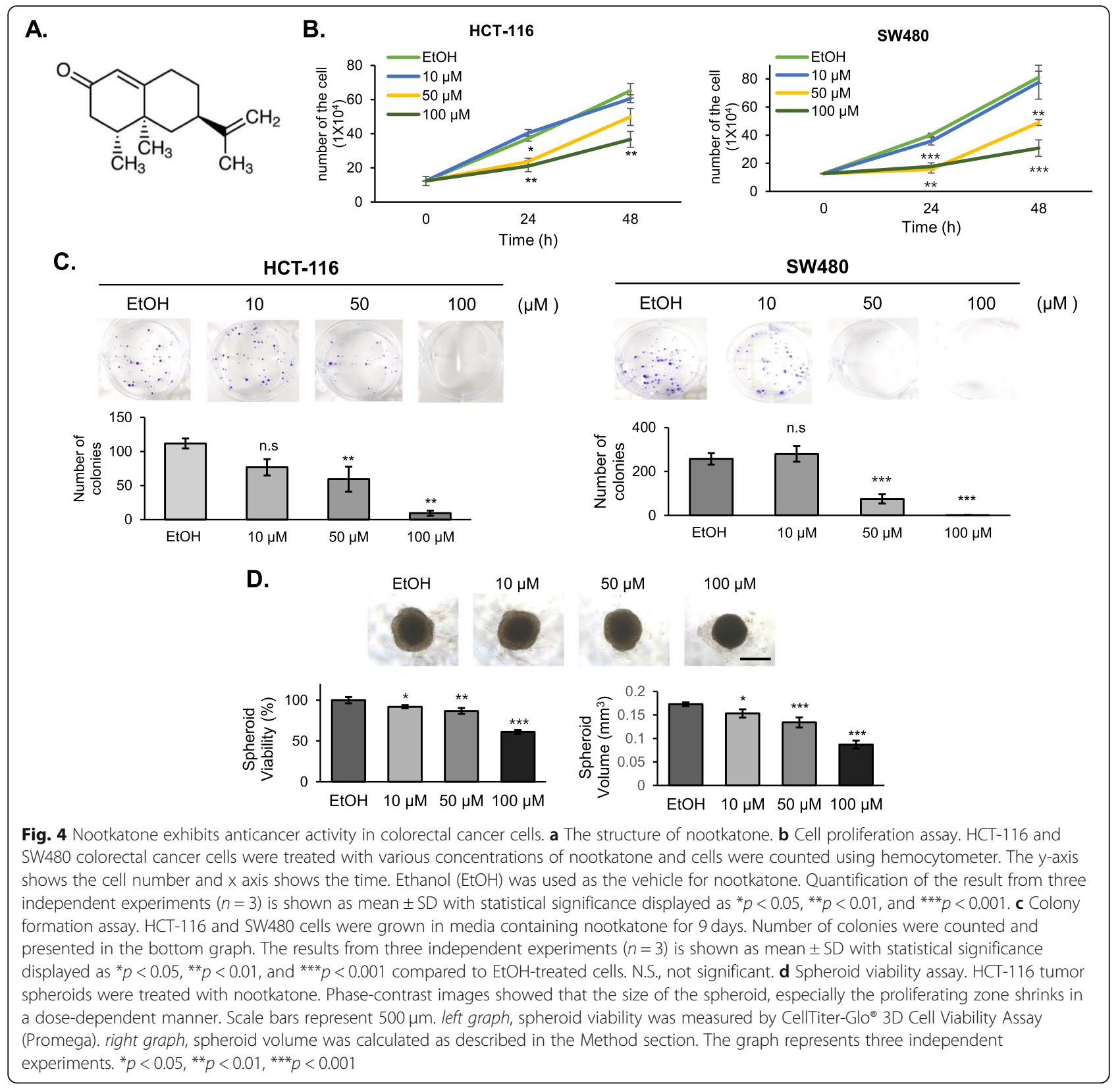

which was the most affected by nootkatone seems to be located within the $133 \mathrm{bp}$ of the NAG-1 promoter (Fig. 6b). It is known that EGR-1 plays a role in transcriptional regulation of NAG-1 in this promoter region [19]. To confirm whether EGR-1 plays a role in nootkatoneinduced NAG-1 expression, an EGR-1 luciferase vector was co-transfected with the $133 \mathrm{bp}$ NAG-1 luciferase construct. The results indicated that EGR-1 indeed increased luciferase activity and activated the $133 \mathrm{bp}$ region of the NAG-1 promoter (Fig. 7c). Subsequently, we measured whether EGR-1 protein was increased by the nootkatone treatment. Nootkatone led to increased EGR-1 expression in both HCT-116 and SW480 cells in a dose-dependent manner (Fig. 7d). Finally, we determined whether nootkatone affects EGR-1 expression at the transcriptional level. The result showed EGR-1 promoter activity was increased in the presence of nootkatone in a dose-dependent manner (Fig. 7e), indicating that nootkatone induces EGR-1 at the transcriptional level ultimately leading to the induction of NAG-1 promoter activity.

\section{Discussion}

Plant extracts and its bioactive compounds have been studied in cancer research. Recent data also suggested the usage of plant derived compounds in new 


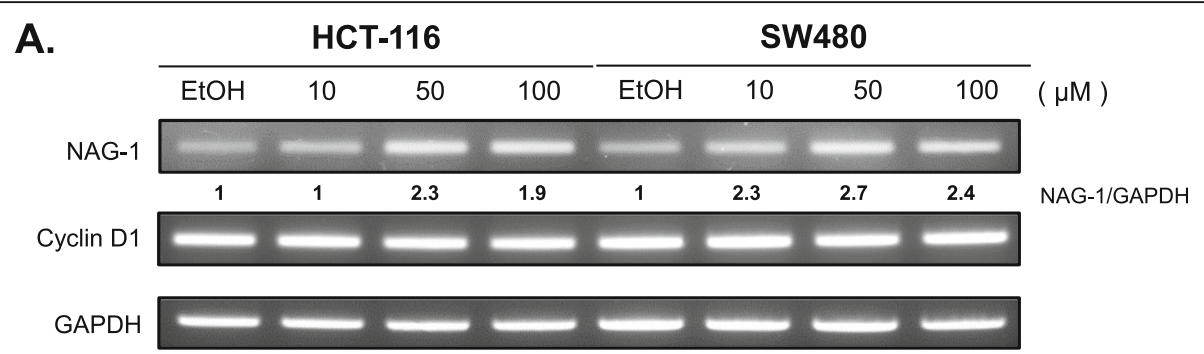

B HCT-116 SW480

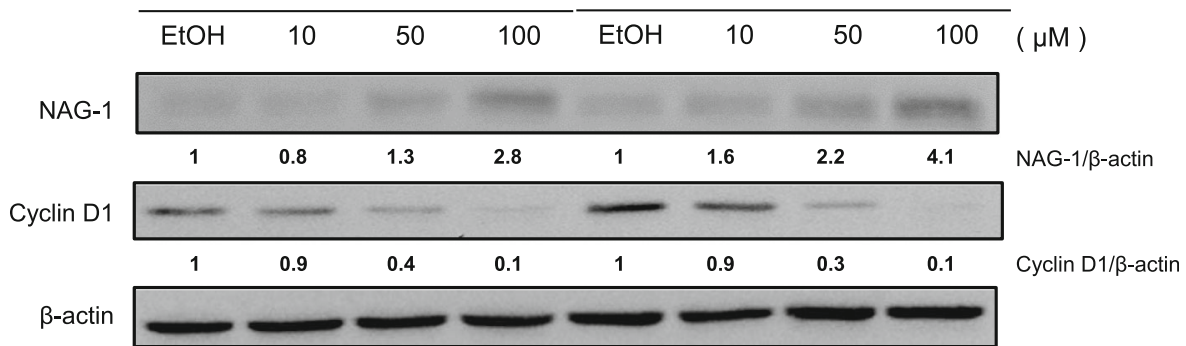

Fig. 5 Nootkatone increases NAG-1 and decreases cyclin D1 expression. a Total RNA was isolated for RT-PCR analysis from nootkatone-treated HCT-116 and SW480 cells. GAPDH was used as housekeeping control gene. NAG-1 RNA levels increased in a dose-dependent manner, whereas expression level of cyclin D1 did not change. b Total proteins were isolated from nootkatone-treated HCT-116 and SW480 cells for western blot analysis. Nooktatone treatment up-regulated NAG-1 protein, while cyclin D1 expression decreased in a dose-dependent manner. $\beta$-actin antibody was used as loading control. The relative expression was determined by the Image J program and represented them in the bottom

generation immunotherapeutic vaccine [24]. A. oxyphylla possesses a wide range of biological activities, including anti-diabetes, anti-liver fibrosis, antidiarrheal, and neuronal protection effects [1]. Moreover, several publications have reported anticancer effects of A. oxyphylla such as in liver cancer cells via AKT pathway suppression [5]. Our findings further confirm its anti-proliferative activity in colorectal cancer cells. Nootkatone is one of nine bioactive compounds found in A. oxyphylla [6], and showed ability to inhibit the expression of inducible nitric oxide synthase (iNOS) and reduce NO production in lipopolysaccharide (LPS) stimulated RAW264.7 cells [25]. Additionally, nootkatone increased survival rates in septic mice by increasing HO-1 expression [25]. Although nootkatone has been linked to anticancer activity in lung cancer via the AMPK pathway [8], the effects of nootkatone against colorectal cancer and its mechanisms underlying these effects remain unknown.

Metastatic cancer encompasses a diverse collection of cells that possess different genetic characteristics and are controlled by many proteins [26-28]. It is also suggested that autophagy plays a role in metastasis [29]. Nootkatone inhibits protein expression that are involved in metastatic cancer and induces autophagy [30]. Thus, molecular elucidation of nootkatone in anti-tumorignesis may lead to better understanding of cancer treatment in metastatic cancer treatment.

In this report, we show that nootkatone treatment contributes to inhibition of cell proliferation in colorectal cancer cells. Our results also suggest that cyclin D1 suppression and NAG-1 induction may at least in part be mechanistically involved in nootkatone-induced antitumorigenic activity.

The suppression of cyclin D1 and induction of NAG-1 by nootkatone were observed at a concentration of $100 \mu \mathrm{M}$. A similar result was reported in lung cancer cells where $100 \mu \mathrm{M}$ of nootkatone was required to obtain cell growth inhibition [8]. Since the systemic concentration of nootkatone and its concentration in tissues has not been reported, and most phytochemicals could reach even higher concentrations in the gastrointestinal (GI) track, the speculation that nootkatone probably reaches $100 \mu \mathrm{M}$ in the GI track is reasonable. Therefore, the large intestinal epithelia, including colon and rectum, could be highlighted as a logical target tissue to further explore nootkatone as an anti-cancer treatment. Additionally, since nootkatone is bio-transformed to various metabolites by fungal strains, the impact of specific nootkatone metabolites may be of particular interest in future cancer studies [31]. In this regard, nootkatone metabolites should be considered as an important 

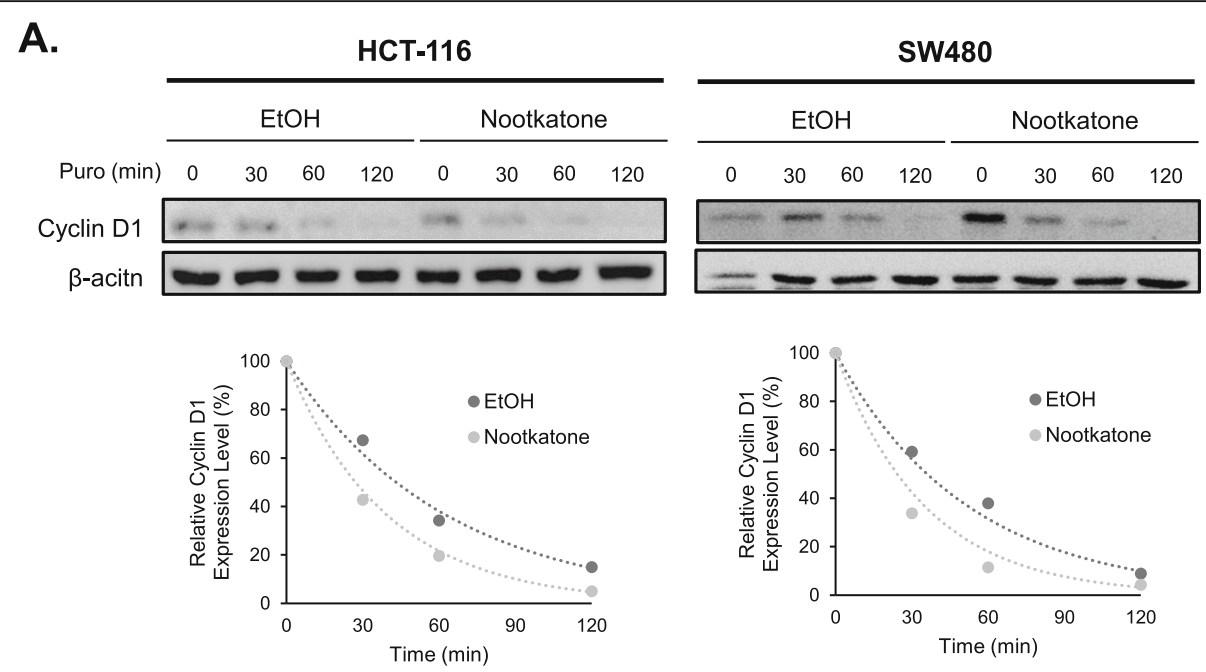

B.

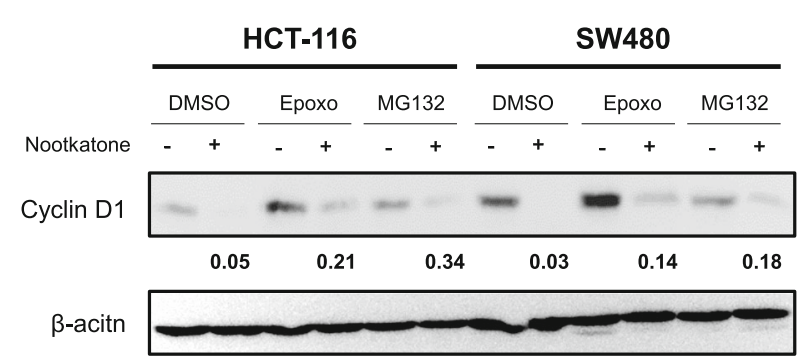

Fig. 6 Nootkatone controls cyclin D1 at the protein level. a HCT-116 and SW480 cells were pre-treated with $100 \mu \mathrm{M}$ of nootkatone for $1 \mathrm{~h}$ and exposed to $20 \mu \mathrm{g} / \mathrm{ml}$ of puromycin (Puro) at different time points. The cell lysate was harvested at each time point, wherein cyclin D1 and $\beta$ actin protein levels were detected. Quantitative analysis was performed by Image J. The bottom graph represents degradation of the cyclin D1 protein over time. $\beta$-actin was used as loading control. $\mathbf{b}$ HCT-116 and SW480 cells were pre-treated with DMSO, $10 \mu \mathrm{M}$ of MG132, or $0.1 \mu \mathrm{M}$ of epoxomicin, followed by treatment with $50 \mu \mathrm{M}$ (HCT-116) or $100 \mu \mathrm{M}$ (SW480) nootkatone for $24 \mathrm{~h}$. $\beta$-actin was used as loading control. Quantitative analysis was performed by Image J

compound with respect to cancer progression. A number of reports suggest that cyclin D1 could be a target for many phytochemicals since cyclin D1 downregulation is common in various phytochemicaltreated samples [32, 33]. Most phytochemicals affect cyclin D1 at the protein level, as was confirmed in the current study regarding nootkatone's effects on cyclin D1 protein. Similarly, DIM, EGCG, damnacanthal, and 6-ginerol downregulates cyclin D1 posttranslationaly, thereby accounting for the antitumorigenic activity of these compounds [16, 32-34]. Cyclin D1 controls many pathways in addition to the cell cycle [35], suggesting that the benefits of cyclin D1 inhibition in cancer may result from several mechanisms.

Transcriptional regulation of NAG-1 is modulated by several cis- and trans-acting elements [12]. The $133 \mathrm{bp}$ promoter region of NAG-1 contains several transcriptional binding sites, including C/EBP $\beta$ [36], p53 [37], EGR-1 [19], and Sp1 [12]. These transcription factors are responsible for the downstream effects of several anticancer compounds including COX inhibitors, PPARy ligands and cancer chemo-preventive agents [38] which increase NAG-1 transcription. Interestingly, sulindac sulfide (or troglitazone)-mediated NAG-1 up-regulation is dependent on the transcription factor EGR-1 in colon cancer cells [39]. EGR-1 binding sites have been detected in the NAG-1 promoter, overlapping the Sp1 binding site. Here, we report that nootkatone increases EGR-1 both at the protein level as well as at the transcriptional level in colorectal cancer cells, and facilitates NAG-1 promoter activity. This suggests that EGR-1 may be responsible for nootkatone -mediated NAG-1 up-regulation. Since PPARy ligand troglitazone also increases EGR-1 expression, we examined whether nootkatone may affect PPARy transcriptional factors. As expected, nootkatone treatment increased PPAR $\gamma$ binding activity as assessed using a reporter construct bearing the PPAR response element (data not shown). Nonetheless, PPAR $\gamma$ activation may not be involved in nootkatone-induced NAG-1 expression at the transcriptional level. Since the emerging pieces of evidence indicate that repurposing of drugs is crucial to the faster 


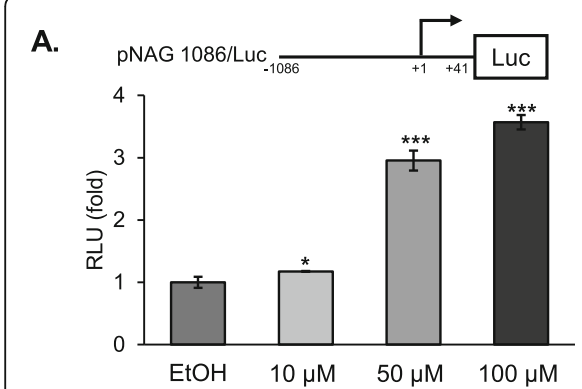

B.

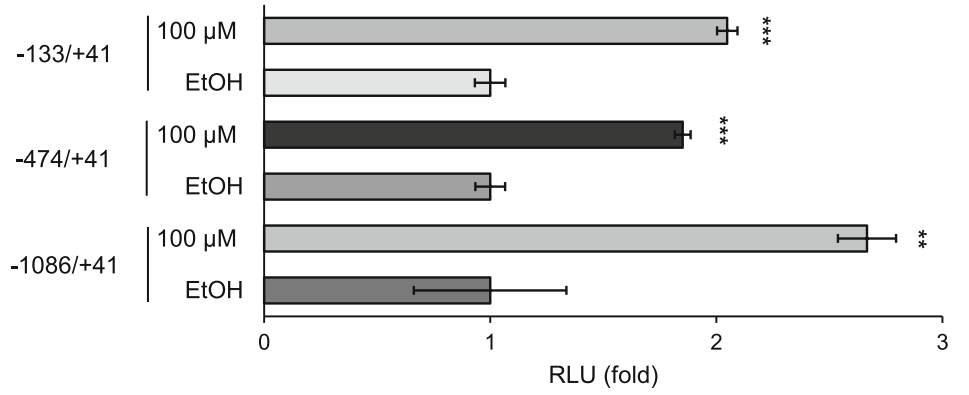

C.
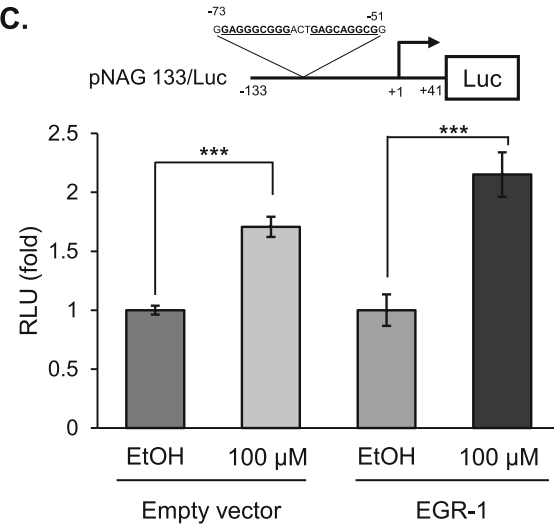

D.

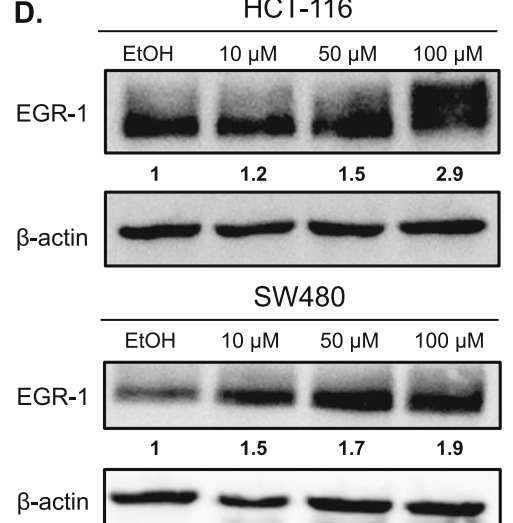

E.
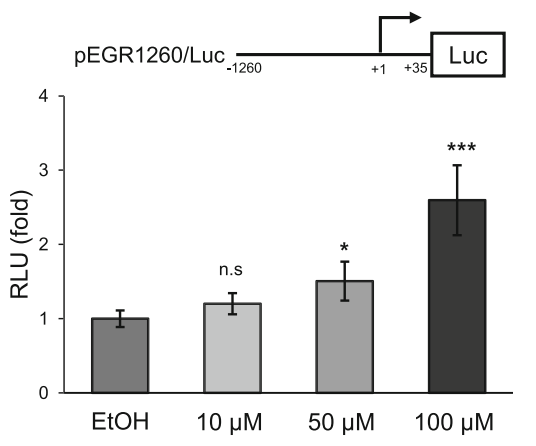

Fig. 7 Nootkatone controls the NAG-1 expression at the transcriptional level. a Nootkatone increases NAG-1 promoter activity. HCT-116 cells were transfected with pNAG-1 - 1086/+ 41 luciferase and pRL-null plasmid. The cells were treated with EtOH or various concentrations of nootkatone for $24 \mathrm{~h}$, and luciferase activity was measured. The $y$-axis refers to the ratio of firefly luciferase over renillar luciferase activity. The EtOH-treated cells were set as 1.0. Statistical significance was displayed as ${ }^{*} p<0.05,{ }^{* *} p<0.001$ versus EtOH-treated cells. The data represent mean \pm SD from three independent experiments. $\mathbf{b}$ Three deletion NAG-1 promoter constructs were co-transfected with pRL-null vector into HCT-116 cells. The cells were treated with $\mathrm{EtOH}$ or $100 \mu \mathrm{M}$ of nootkatone for $24 \mathrm{~h}$, and luciferase activity was measured. Fold induction refers to the ratio of luciferase activity in nootkatone-treated cells versus EtOH-treated cells. Statistical significance was displayed as ${ }^{* *} p<0.01$ and ${ }^{* *} p<0.001$ versus EtOH-treated cells. The data represent mean \pm SD from three independent experiments. $\mathbf{c}$ HCT-116 cells were co-transfected with wild type pNAG-1 - 133/+ 41 in the presence of empty or EGR-1 expression vector. Cells were subsequently treated with $100 \mu \mathrm{M}$ nootkatone for $24 \mathrm{~h}$. The results are presented as means \pm S.D. of three independent transfections. $\mathbf{d}$ Western blot of EGR-1 protein in the presence of nootkatone. $\beta$-actin was used as loading control. e Luciferase activity of EGR-1 promoter-luciferase construct (pEGR-1260-LUC). The cells were treated with EtOH or nootkatone for $24 \mathrm{~h}$ prior to measurement of luciferase activity. Fold induction refers to the ratio of luciferase activity in nootkatone-treated cells compared to EtOH-treated cells. Statistical significance represented as ${ }^{*} p<0.05,{ }^{* * *} p<0.001$ versus EtOH-treated cells. n.s. represents not significant. The data represent mean \pm SD from four independent experiments

and cheaper discovery of anti-cancerous drugs [40], nootkatone should be seriously considered for the design of future cancer drugs.

\section{Conclusions}

Our results indicate that A. oxyphylla and its bioactive compound nootkatone exhibit antiproliferative activity in colorectal cancer cells. NAG-1 induction and cyclin D1 downregulation may contribute at least in part to the antiproliferative activity of nootkatone.

\section{Abbreviations}

NAG-1: Nonsteroidal anti-inflammatory drug activated gene-1;

GDF15: Growth differentiation factor 15; EGR-1: Early Growth Response 1; PTEN: Phosphatase and tensin homolog; PPARY: Peroxisome proliferatoractivated receptor gamma; ABTS: 2,2'-Azino-bis(3-ethylbenzothiazoline-6sulfonic acid); DPPH: 2,2-diphenyl-1-picrylhydrazyl; C/EBPß: CCAAT/enhancerbinding protein beta; DIM: 3-3'-di-indolymethane; EGCG: Epigallocatechin gallate; Sp1: Specificity protein 1; COX: Cyclooxygenase; HO-1: Heme Oxygenase-1

\section{Acknowledgements}

We thank Hyunjin Moon and Yukyung Hong from Department of Veterinary Medicine, College of Veterinary Medicine, Seoul National University for their technical supports.

\section{Authors' contributions}

EY and SJB designed the study. EY, JL, PL, CK, and EP performed the experiments. EY, JL, PL, JR, and $S J B$ analyzed, interpreted the data and wrote the article. All authors have approved this submission.

\section{Funding}

This work was supported by the Research Institute for Veterinary Science, and BK21 PLUS Program for Creative Veterinary Science Research Center, Seoul National University, and by a National Research Foundation of Korea (NRF) grant funded by the Korean government (2018R1A2B2002923) to S.J.B. This work was also partially supported by a clinical research grant (NCC1810150) provided by the National Cancer Center to J.R. and S.J.B. The 
funding agency did not have any influence in the design of the study and collection, analysis, and interpretation of data and in writing the manuscript.

\section{Availability of data and materials}

The materials during the current study are available from the corresponding author on reasonable request.

\section{Ethics approval and consent to participate}

Not Applicable.

\section{Consent for publication}

Not Applicable.

\section{Competing interests}

The authors declare that there is no conflict of interest.

\section{Author details}

'Department of Veterinary Medicine, College of Veterinary Medicine and Research Institute for Veterinary Science, Seoul National University, Seoul 08826, South Korea. ${ }^{2}$ Department of Otolaryngology-Head and Neck Surgery, Research Institute and Hospital, National Cancer Center, Goyang, South Korea. ${ }^{3}$ R\&D Center, EastHill Co. 33, Omokcheon-ro 132 beon-gil, Gwonseon-gu, Suwon-si, Gyeonggi-do 16642, South Korea.

Received: 2 July 2020 Accepted: 3 September 2020 Published online: 14 September 2020

\section{References}

1. Zhang Q, Zheng Y, Hu X, Hu X, Lv W, Lv D, Chen J, Wu M, Song Q, Shentu J. Ethnopharmacological uses, phytochemistry, biological activities, and therapeutic applications of Alpinia oxyphylla Miquel: a review. J Ethnopharmacol. 2018:224:149-68.

2. Xie Y, Xiao M, Li D, Liu H, Yun F, Wei Y, Sang S, Du G. Anti-diabetic effect of Alpinia oxyphylla extract on 57BL/KsJ db-/db- mice. Exp Ther Med. 2017; 13(4):1321-8.

3. Balta C, Ciceu A, Herman H, Rosu M, Boldura O-M, Hermenean A. Dosedependent Antifibrotic effect of Chrysin on regression of liver fibrosis: the role in extracellular matrix remodeling. Dose-Response. 2018;16: 155932581878983

4. Wang S, Zhao Y, Zhang J, Huang X, Wang Y, Xu X, Zheng B, Zhou X, Tian H, Mei Q. Antidiarrheal effect of Alpinia oxyphylla Miq. (Zingiberaceae) in experimental mice and its possible mechanism of action. J Ethnopharmacol. 2015:168:182-90

5. Hui F, Qin X, Zhang Q, Li R, Liu M, Tianshu R, Zhao M, Zhao Q. Alpinia oxyphylla oil induces apoptosis of hepatocellular carcinoma cells via PI3K Akt pathway in vitro and in vivo. Biomed Pharmacother. 2019;109:2365-74.

6. Li Y-H, Chen F, Wang J-F, Wang Y, Zhang J-Q, Guo T. Analysis of nine compounds from Alpinia oxyphylla fruit at different harvest time using UFLC-MS/MS and an extraction method optimized by orthogonal design. Chem Cent J. 2013;7:134

7. Choi H-J, Lee J-H, Jung Y-S. (+)-Nootkatone inhibits tumor necrosis factor a/ interferon $y$-induced production of chemokines in $\mathrm{HaCaT}$ cells. Biochem Biophys Res Commun. 2014:447(2):278-84

8. Hung LVM, Moon JY, Ryu J-y, Cho SK. Nootkatone, an AMPK activator derived from grapefruit, inhibits KRAS downstream pathway and sensitizes non-small-cell lung cancer A549 cells to adriamycin. Phytomedicine. 2019; 63:153000.

9. Oi $Y$, Cheng $X$, Jing $H$, Yan $T$, Xiao $F$, Wu B, Bi K, Jia Y. Combination of schisandrin and nootkatone exerts neuroprotective effect in Alzheimer's disease mice model. Metab Brain Dis. 2019;34(6):1689-703.

10. Lin RJ, Yen CM, Chou TH, Chiang FY, Wang GH, Tseng YP, Wang L, Huang TW, Wang HC, Chan LP, et al. Antioxidant, anti-adipocyte differentiation, antitumor activity and anthelmintic activities against Anisakis simplex and Hymenolepis nana of yakuchinone a from Alpinia oxyphylla. BMC Complement Altern Med. 2013:13:237

11. Chun KS, Park KK, Lee J, Kang M, Surh YJ. Inhibition of mouse skin tumor promotion by anti-inflammatory diarylheptanoids derived from Alpinia oxyphylla Miquel (Zingiberaceae). Oncol Res. 2002;13(1):37-45.

12. Baek SJ, Horowitz JM, Eling TE. Molecular cloning and characterization of human nonsteroidal anti-inflammatory drug-activated gene promoter.
Basal transcription is mediated by Sp1 and Sp3. J Biol Chem. 2001; 276(36):33384-92

13. Baek SJ, Kim KS, Nixon JB, Wilson LC, Eling TE. Cyclooxygenase inhibitors regulate the expression of a TGF-beta superfamily member that has proapoptotic and antitumorigenic activities. Mol Pharmacol. 2001;59(4):901-8.

14. Baek SJ, Okazaki R, Lee SH, Martinez J, Kim JS, Yamaguchi K, Mishina Y, Martin DW, Shoieb A, McEntee MF, et al. Nonsteroidal anti-inflammatory drug-activated gene-1 over expression in transgenic mice suppresses intestinal neoplasia. Gastroenterology. 2006;131(5):1553-60.

15. Jirawatnotai $S, H u$ Y, Michowski W, Elias JE, Becks L, Bienvenu F, Zagozdzon A, Goswami T, Wang YE, Clark AB, et al. A function for cyclin D1 in DNA repair uncovered by protein interactome analyses in human cancers. Nature. 2011:474(7350):230-4

16. Lee SH, Cekanova M, Baek SJ. Multiple mechanisms are involved in 6gingerol-induced cell growth arrest and apoptosis in human colorectal cancer cells. Mol Carcinog. 2008:47(3):197-208.

17. Nair P, Muthukkumar S, Sells SF, Han SS, Sukhatme VP, Rangnekar VM. Early growth response-1-dependent apoptosis is mediated by p53. J Biol Chem. 1997;272(32):20131-8.

18. Virolle T, Adamson ED, Baron V, Birle D, Mercola D, Mustelin T, de Belle I. The Egr-1 transcription factor directly activates PTEN during irradiationinduced signalling. Nat Cell Biol. 2001;3(12):1124-8.

19. Baek SJ, Kim JS, Moore SM, Lee SH, Martinez J, Eling TE. Cyclooxygenase inhibitors induce the expression of the tumor suppressor gene EGR-1, which results in the up-regulation of NAG-1, an antitumorigenic protein. Mol Pharmacol. 2005;67(2):356-64.

20. Wilmanski T, Zhou X, Zheng W, Shinde A, Donkin SS, Wendt M, Burgess JR, Teegarden D. Inhibition of pyruvate carboxylase by 1a,25-dihydroxyvitamin D promotes oxidative stress in early breast cancer progression. Cancer Lett. 2017:411:171-81.

21. Lee J, Kim I, Yoo E, Baek SJ. Competitive inhibition by NAG-1/GDF-15 NLS peptide enhances its anti-cancer activity. Biochem Biophys Res Commun. 2019:519(1):29-34

22. Prasansuklab A, Tencomnao T. Acanthus ebracteatus leaf extract provides neuronal cell protection against oxidative stress injury induced by glutamate. BMC Complem Altern Med. 2018;18(1):278.

23. Kirtonia A, Sethi G, Garg M. The multifaceted role of reactive oxygen species in tumorigenesis. Cell Mol Life Sci. 2020. https://doi.org/10.1007/s00018-020-03536-5.

24. Gupta B, Sadaria D, Warrier VU, Kirtonia A, Kant R, Awasthi A, Baligar P, Pal JK, Yuba E, Sethi G, et al. Plant lectins and their usage in preparing targeted nanovaccines for cancer immunotherapy. Semin Cancer Biol. 2020. In Press.

25. Tsoyi K, Jang HJ, Lee YS, Kim YM, Kim HJ, Seo HG, Lee JH, Kwak JH, Lee D-U, Chang KC. (+)-Nootkatone and (+)-valencene from rhizomes of Cyperus rotundus increase survival rates in septic mice due to heme oxygenase-1 induction. J Ethnopharmacol. 2011:137(3):1311-7.

26. Shinde A, Paez JS, Libring S, Hopkins K, Solorio L, Wendt MK. Transglutaminase-2 facilitates extracellular vesicle-mediated establishment of the metastatic niche. Oncogenesis. 2020;9(2):16

27. Libring S, Shinde A, Chanda MK, Nuru M, George H, Saleh AM, Abdullah A, Kinzer-Ursem TL, Calve S, Wendt MK, et al. The dynamic relationship of breast Cancer cells and fibroblasts in Fibronectin accumulation at primary and metastatic tumor sites. Cancers. 2020;12(5):1270.

28. Shinde A, Libring S, Alpsoy A, Abdullah A, Schaber JA, Solorio L, Wendt MK Autocrine Fibronectin inhibits breast Cancer metastasis. Mol Cancer Res. 2018;16(10):1579-89.

29. Shinde A, Hardy SD, Kim D, Akhand SS, Jolly MK, Wang W-H, Anderson JC, Khodadadi RB, Brown WS, George JT, et al. Spleen tyrosine kinase-mediated autophagy is required for epithelial-Mesenchymal plasticity and metastasis in breast Cancer. Cancer Res. 2019;79(8):1831-43.

30. Zhu X, Li X, Chen Z. Inhibition of anticancer growth in retinoblastoma cells by naturally occurring sesquiterpene nootkatone is mediated via autophagy, endogenous ROS production, cell cycle arrest and inhibition of NF-KB signalling pathway. J BUON. 2020;25(1):427-31

31. Gliszczyńska A, Łysek A, Janeczko T, Świtalska M, Wietrzyk J, Wawrzeńczyk C. Microbial transformation of (+)-nootkatone and the antiproliferative activity of its metabolites. Bioorg Med Chem. 2011:19(7):2464-9.

32. Zhang X, Sukamporn P, Zhang S, Min KW, Baek SJ. 3,3'-diindolylmethane downregulates cyclin D1 through triggering endoplasmic reticulum stress in colorectal cancer cells. Oncol Rep. 2017;38(1):569-74. 
33. Sukamporn P, Rojanapanthu P, Silva G, Zhang X, Gritsanapan W, Baek SJ Damnacanthal and its nanoformulation exhibit anti-cancer activity via cyclin D1 down-regulation. Life Sci. 2016;152:60-6.

34. Zhang X, Min K-W, Wimalasena J, Baek SJ. Cyclin D1 degradation and p21 induction contribute to growth inhibition of colorectal cancer cells induced by epigallocatechin-3-gallate. J Cancer Res Clin Oncol. 2012;138(12):2051-60.

35. Qie S, Diehl JA. Cyclin D1, cancer progression, and opportunities in cancer treatment. Int J Mol Med. 2016;94(12):1313-26.

36. Nualsanit T, Rojanapanthu P, Gritsanapan W, Lee SH, Lawson D, Baek SJ. Damnacanthal, a noni component, exhibits antitumorigenic activity in human colorectal cancer cells. J Nutr Biochem. 2012;23(8):915-23.

37. Wilson LC, Baek SJ, Call A, Eling TE. Nonsteroidal anti-inflammatory drugactivated gene (NAG-1) is induced by genistein through the expression of p53 in colorectal cancer cells. Int J Cancer. 2003;105(6):747-53.

38. Baek SJ, Eling T. Growth differentiation factor 15 (GDF15): a survival protein with therapeutic potential in metabolic diseases. Pharmacol Ther. 2019;198:46-58.

39. Baek SJ, Wilson LC, Hsi LC, Eling TE. Troglitazone, a peroxisome proliferatoractivated receptor gamma (PPAR gamma) ligand, selectively induces the early growth response-1 gene independently of PPAR gamma. A novel mechanism for its anti-tumorigenic activity. J Biol Chem. 2003;278(8):5845-53.

40. Kirtonia A, Gala K, Fernandes SG, Pandya G, Pandey AK, Sethi G, Khattar E, Garg M. Repurposing of drugs: an attractive pharmacological strategy for cancer therapeutics. Semin Cancer Biol. 2020;S1044-579X(20):30094-8.

\section{Publisher's Note}

Springer Nature remains neutral with regard to jurisdictional claims in published maps and institutional affiliations.

Ready to submit your research? Choose BMC and benefit from:

- fast, convenient online submission

- thorough peer review by experienced researchers in your field

- rapid publication on acceptance

- support for research data, including large and complex data types

- gold Open Access which fosters wider collaboration and increased citations

- maximum visibility for your research: over $100 \mathrm{M}$ website views per year

At $\mathrm{BMC}$, research is always in progress.

Learn more biomedcentral.com/submissions 